\title{
Malaria vaccine in sight?
}

\section{The latest development in the molecular biology of malaria parasites is not so much a promise of prophylaxis as a sign that the promise will not forever be postponed.}

WHAT a day to meet! Thus Dr Adetokumbo Lucas, director of the World Health Organization's Special Programme for Research and Training in Tropical Diseases, brandishing one day last week photocopies of a set of page proofs of the articles in the current issue of Science (10 August) describing the structure of the gene for the principal antigen of the sporozoite form of human malaria, caused by the parasite Plasmodium falciparum. What Lucas meant was that since Nussenzweig and his associates at New York University last year described the structure of the corresponding antigen of monkey malaria (Nature 305, 29; 1983), everybody has been waiting for the other shoe to fall. And now it has.

As always, even the expected is surprising. Of the work now published, the more complete is that reported from the National Institute of Allergy and Infectious Diseases, the National Cancer Institute and the Walter Reed Army Institute of Research in the United States (J.B. Dame et al., Science 225, 593; 1984), but the New York University (NYU) group (V. Enea $e t$ al., Science 225,$628 ; 1984$ ) concurs in the chief finding - that the most conspicuous feature of the circumsporozoite (CS) protein from $P$. falciparum is a long stretch in which a sequence of four amino acids is repeated end to end. Repetition on this pattern marks out the structure of the two CS molecules whose structure has previously been inferred (from nucleotide sequences) - those of $P$. knowlesi and $P$. cynomolgi. But there the repeating units are respectively 12 and 11 amino acids long. That the repeating units in the antigenic coat of $P$. falciparum should be so short is one surprise. There will now be a great rush to tell what can be said of the presumed repetitive amino acid sequences in other species of Plasmodium, causing malaria in people and animals.

As now described by Dame et al., the CS gene of $P$. falciparum is in three sections a beginning, a middle and an end. The characteristic repeating section is the middle, which consists of no less than 41 four-codon repeats, corresponding to the amino acid sequence asparagine-alanine-asparagine-proline. But four of the 41 repeating units have a different structure, corresponding to the tetrapeptides asparagine-valine-aspartic acid-proline. In outline, the structure of the CS gene of $P$. knowlesi is similar but shorter, although the middle repeating unit is shorter, at 12 twelve-codon units.

The beginning and the end of both genes differ from the central repeating stretch in carrying codons that would specify hydrophobic amino acids. The downstream end is assumed to be anchored in the outer cell membrane of the malaria parasite. Perhaps in real life, individual malaria parasites are protected from the outside world by sheaths of protein molecules whose stability depends not only on the way in which the molecules are anchored in the membrane but also on their sideways interaction with each other. It remains to be seen whether the model-builders will justify their existence by offering an explanation of what the three different repetitive nucleotide units now defined for malarial parasites imply about the presumed common function of the corresponding amino acid sequences. It must also be significant, but of something as yet unknown, that the repeating gene sequence is preceded and followed, both in $P$. falciparum and $P$. knowlesi, by closely similar regions (15 and 12 amino acids long respectively) whose similarity suggests a structural function to be identified.

Evolutionists will also prick up their ears at these developments. The conventional wisdom is that parasites such as Plasmodium are so exquisitely adapted to their hosts - replicating at their expense without utterly destroying them - that their evolution must have gone hand in hand with at least that of the primates. One minor issue is partly answered by Dame et al.,: a gene with 42 four-codon repeats should be prone to instability in the sense that genetic recombination, insensitive as it is to anything but the chemistry of codons, will often decrease or increase the number of repetitive elements in a nucleotide sequence. But apart from the four odd units in the gene of $\boldsymbol{P}$. falciparum described by Dame et al., there are also 19 regular repeating units in which different nucleotide sequences code for the same amino acid sequence. Recombination has its hands tied behind its back. The NYU group says that the strain of $\boldsymbol{P}$. falciparum they have investigated has a stretch of 23 identical repeating units, but there is no room for such a structure in the gene that Dame et al. describe. So is it possible that the details of the fourfold repetition are strain-specific? This appears to be the case with the blood stage antigen (Coppel et al., Nature 306, 751; 1984).

Much will hang on the answer to that question (which should be easily obtained). The potential importance of the discoveries now described depends to a large extent on observations that quite small portions of the CS proteins, hardly more than a dozen amino acids, are antigenic. So the great hope evoked by this latest development is that it may be possible to make vaccines against malaria by synthesizing an appropriate polypeptide. The snag is that if interstrain variability is too flamboyant, the cocktail needed may be impossibly complicated. The immunogenic capacities of the nearly identical sequences before and after the repetitive sequences therefore cry out for study.

So how long will it be before there is a vaccine against malaria? The Lucases of this world know a trick question when they are asked one. The only proper answer, anyway, is "sometime in some future decade". Astonishing though it may seem, although malaria may be one of the bestdescribed diseases, next to nothing is known, in modern language, of the mechanism of the details of the immune defence against it. Are antibodies, and the B-lymphocytes that secrete them, a sufficient defence against invasion by the sporozoites whose antigens are now described? How, artificially, will it be possible to give people a degree of protection against the infective form of the malaria parasite that will prevent lodgement in the liver? Is the cocktail-vaccine eventually needed bound to be one that caters not only for the antigenic variability of the invasive sporozoites but for the merozoites (matured in the liver) which eventually infect the red blood cells? And how, at the end of the cycle, can one prevent the maturation of the gametocytes from red blood cells, so breaking the cycle of infection via the mosquito?

Nobody knows. So we, but especially Dr Lucas, are in a cleft stick. In the endless battle against malaria, well-documented since Roman times and now said by the World Health Organization to be responsible for 150 million new cases a year (for want of an accurate number), we know that each new discovery may prove to be the basis for effective prophylaxis but with a chance of, say, one in 20 . The work of Dame et al., supported by the Nussenzweigs, is one of those 20. Time will tell whether discovery will be crucial. But as a pointer to the questions that need next to be answered, its value is already clear.

John Maddox 